INRA Prod. Anim. 1994,7 (5), 315 - 326.

\section{P. CHEMINEAU, J.A. DELGADILLO*}

INRA Physiologie de la Reproduction 37380 Nouzilly

* Universidad Autonoma Agraria Antonio Narro, Carretera a Sta Fé y Periférico Apartado Postal 940, Torreon, Coahuila, México

\title{
Neuroendocrinologie de la reproduction chez les caprins
}

\begin{abstract}
L'équilibre des mécanismes contrôlant la reproduction repose sur une relation permanente entre le système nerveux central et les gonades, relation assurée par les hormones gonadotropes et stéroïdiennes. Mais différents facteurs externes interviennent pour modifier cet équilibre, notamment la saison pour l'espèce caprine.
\end{abstract}

Dans les deux sexes, le système nerveux central, par l'intermédiaire de la LH-RH (Luteinizing Hormone-Releasing Hormone, ou $\mathrm{GnRH}$ ), commande l'antéhypophyse qui, à son tour, sécrète les hormones gonadotropes LH

\section{Résumé}

L'hypothalamus, par l'intermédiaire de la sécrétion du LH-RH, commande la libération épisodique des hormones gonadotropes LH et FSH dans la circulation générale. La LH n'est pas sécrétée de façon continue par l'hypohyse mais sous forme de "pulses", définis par leur fréquence et leur amplitude, qui stimulent la libération de la testostérone par le testicule chez le mâle et de l'oestradiol et de la progestérone par l'ovaire chez la femelle. La FSH est sécrétée de façon moins épisodique. Chez la chèvre, la modulation des rétroactions négatives et positives de la progestérone et de l'oestradiol sur l'axe hypothalamo-hypohysaire joue un rôle clé dans la régulation des différents événements du cycle oestrien. Une imprégnation par la progestérone n'est pas nécessaire pour l'induction du comportement d'oestrus par l'oestradiol.

Dans les deux sexes, le saisonnement de l'activité neuroendocrinienne est responsable des fortes variations saisonnières d'activité sexuelle. Un accroissement de la rétroaction négative de l'oestradiol sur l'axe hypothalamo-hypohysaire est responsable de la faible activité gonadotrope pendant la saison d'anoestrus. Cet effet est sous la commande des variations photopériodiques qui agissent sur le système nerveux central par l'intermédiaire de la modification de la durée de sécrétion de la mélatonine nocturne. Les changements dans le régime alimentaire provoquent également des variations dans l'activité gonadotrope.

La mise en présence des femelles avec le bouc provoque une augmentation immédiate de la fréquence des pulses de LH chez celles-ci.
(Luteinizing Hormone) et FSH (Follicule Stimulating Hormone), directement responsables de la stimulation des gonades (testicule et ovaire). Celles-ci sont le lieu de synthèse et de sécrétion des hormones stéroïdes dont les rôles sont multiples. Les stéroïdes participent de façon importante au fonctionnement de la spermatogénèse et de la folliculogénèse, à l'apparition et au maintien du comportement sexuel et au développement des caractères sexuels secondaires. Ils exercent également un rétro-contrôle, tantôt négatif, tantôt positif, sur l'axe hypothalamo-hypohysaire, prévenant, par là, un "emballement" du système (figure 1). Les équilibres et les relations existant entre ces différentes substances hormonales conditionnent le déroulement temporel de l'activité sexuelle des mâles (spermatogénèse et comportement sexuel) et des femelles (cycles œstriens et ovariens).

Plusieurs facteurs sont susceptibles de modifier cet équilibre : la saison, le niveau d'alimentation et la présence de partenaires sexuels.

L'ensemble de ces mécanismes est bien connu chez plusieurs espèces de mammiferres domestiques, notamment chez les ovins. Nous nous attacherons ici à décrire ces mécanismes, lorsqu'ils sont connus, chez les caprins, et éga- 
Figure 1. Relations entre les facteurs de l'environnement, le système nerveux central, l'hypohyse et les gonades dans l'espèce caprine.

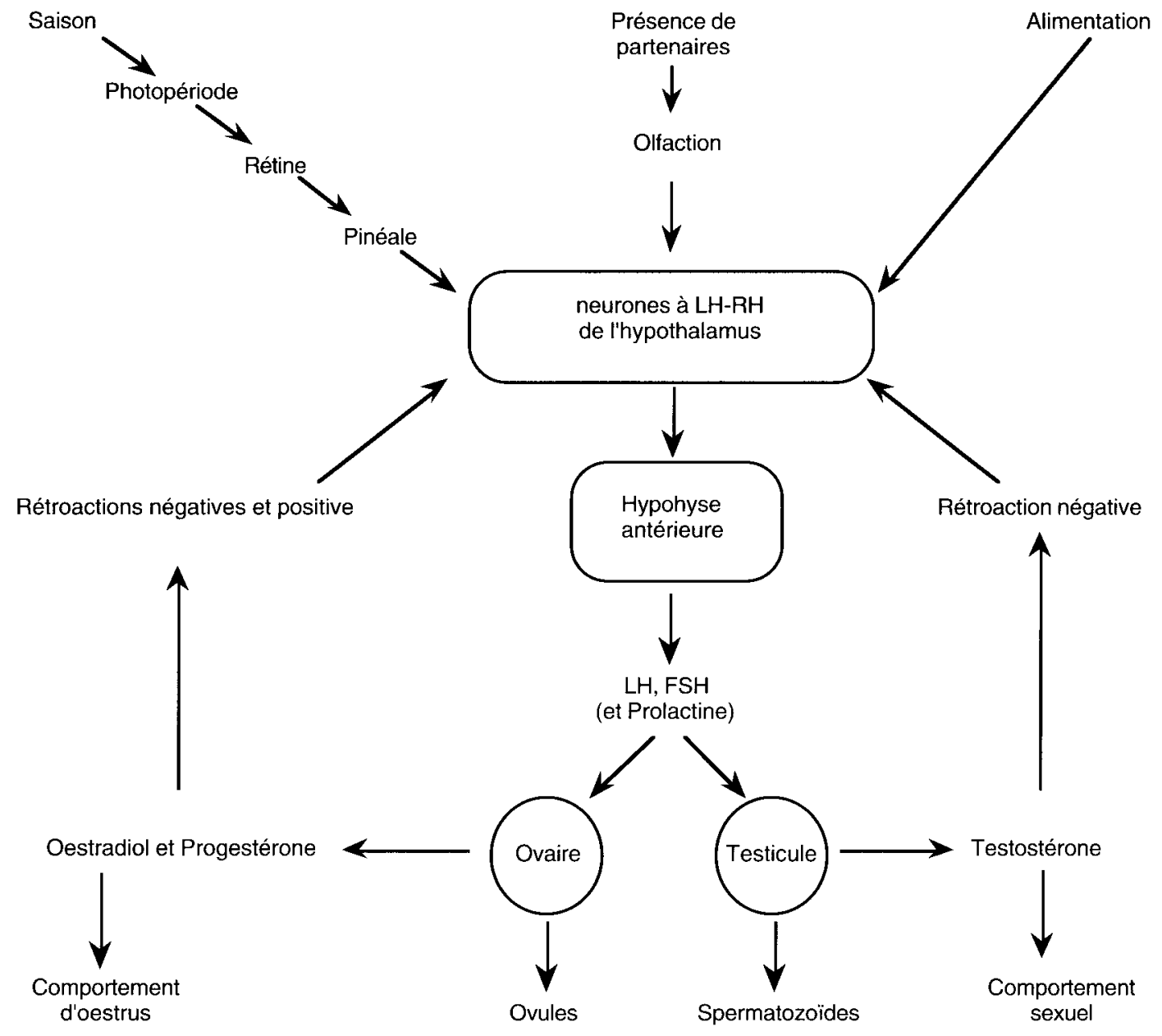

lement à mettre en évidence les particularités de cette espèce, lorsqu'elles existent.

\section{1 / Activité neuroendocrinienne du mâle}

Chez le bouc, l'activité spermatogénétique (= ensemble des processus qui conduisent à la fabrication des spermatozoïdes) est sous la dépendance de la LH et de la FSH. Celles-ci participent non seulement à la différenciation et à la multiplication des cellules germinales, mais également à la synthèse et la sécrétion de la testostérone par les cellules de Leydig du testicule. La testostérone participe au maintien de la spermatogénèse, et déclenche également le comportement sexuel et exerce un rétrocontrôle sur les hormones gonadotropes.

La prolactine joue un rôle plus incertain dans la reproduction. Elle peut cependant être considérée comme un bon indicateur de la perception de la durée du jour.

\section{1 / Sécrétion de LH, FSH et testostérone}

La LH n'est pas libérée de façon continue par l'hypophyse. De brusques épisodes sécrétoires, commandés par l'activité des neurones à LH-RH de l'hypothalamus, alternent avec des périodes de repos pendant lesquelles une sécrétion basale est enregistrée. Ces épisodes sécrétoires, appelés "pulses" sont caractérisés par leur amplitude, directement reliée à la quantité de LH libérée dans la circulation générale (figure 2). Après la fin de la libération, la décroissance progressive dans le sang représente le temps nécessaire à la disparition de l'hormone de la circulation sanguine (plusieurs dizaines de minutes). L'étude de la sécrétion rapide de $\mathrm{LH}$ dans différentes races (Alpine, Créole) et différentes situations physiologiques (saison ou contre-saison sexuelle), semble indiquer que la fréquence des pulses (nombre de pulses en un nombre d'heures donné) est au moins aussi importante que leur amplitude pour déterminer la réponse des 
Figure 2. Variations rapides des teneurs plasmatiques en $\mathrm{LH}$ et testostérone chez un bouc Créole prélevé toutes les 20 minutes (Chemineau et al, non publié).

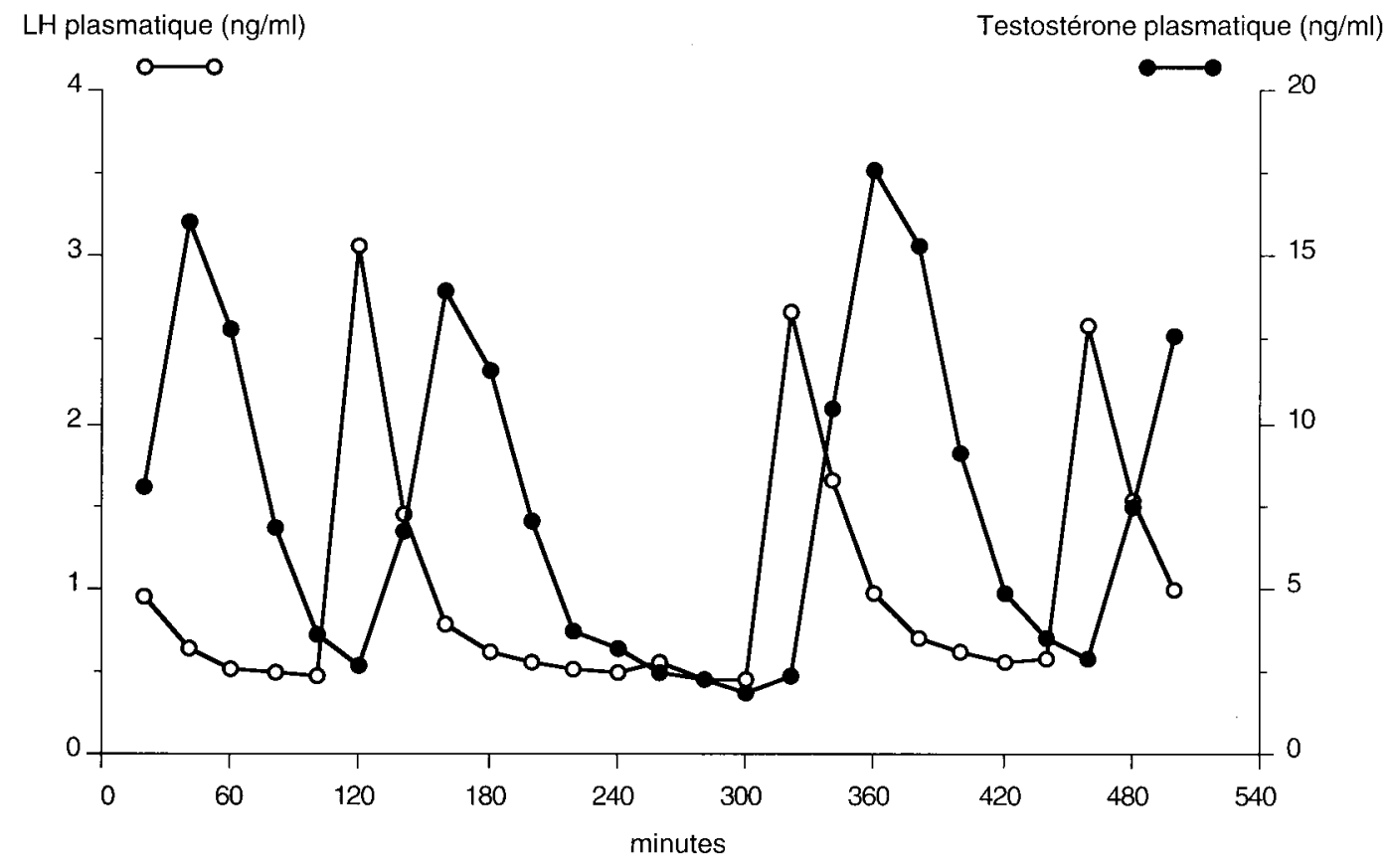

gonades (Muduuli et al 1979, Delgadillo et Chemineau 1992).

Ces brusques changements de la concentration plasmatique de LH entraînent une stimulation rapide des cellules de Leydig du testicule qui répondent en libérant la testostérone dans le sang (figure 2). Chaque pulse de LH est donc suivi d'un pulse de testostérone dont l'amplitude varie selon la situation physiologique du mâle. La vitesse de disparition de la testostérone dans le sang est plus lente que celle de la LH. Lorsque la fréquence des pulses n'est pas très élevée, la testostérone revient à son niveau de base entre deux pulses.

La FSH est sécrétée d'une manière plus complexe que la LH. Même s'il est possible d'identifier quelques pulses dans une série chronologique, la sécrétion de FSH semble continue plutôt qu'épisodique (Muduuli et al 1979).

\section{2 / Variations saisonnières de l'activité neuroendocrinienne}

Dans les races saisonnées, l'alternance de périodes d'activité et d'inactivité sexuelle est d'origine centrale. Ce sont les variations de l'activité gonadotrope qui sont responsables de la faible activité au printemps et en été et, inversement, de l'intense activité en automne et en hiver.

Chez le bouc Alpin, le niveau de base de LH $(0,3 \mathrm{ng} / \mathrm{ml}$ de plasma $)$, la fréquence des pulses (environ 1 en 8 heures), leur amplitude (moins de $0,2 \mathrm{ng} / \mathrm{ml}$ ) et donc la concentration moyenne de LH $(0,4 \mathrm{ng} / \mathrm{ml}$ de plasma), sont faibles de janvier à mai (figure $3 a, 3 b$ ). L'amplitude des pulses augmente régulièrement en juin et juillet pour atteindre $1,0 \mathrm{ng} / \mathrm{ml}$ en août. Puis, en septembre, leur fréquence augmente brusquement (3,5 pulses en 8 heures), tandis que leur amplitude diminue, à cause de la relation inverse entre fréquence et amplitude, mais aussi probablement sous l'influence de la testostérone sécrétée alors en grandes quantités ( $4 \mathrm{ng} / \mathrm{ml}$ de plasma en août, $13 \mathrm{ng} / \mathrm{ml}$ en septembre) (figure 3c). Après les niveaux élevés de LH et de testostérone en août et en septembre, une diminution progressive est observée jusqu'en janvier, puis le cycle annuel recommence (Saumande et Rouger 1972, Delgadillo et Chemineau 1992). Des observations similaires sont rapportées pour le bouc Cashmere australien, avec 6 mois de décalage, dans l'hémisphère sud (Walkden-Brown 1991).

Ainsi que l'indiquent les concentrations plasmatiques de testostérone, mesurées une fois par semaine pendant deux ans sur les mêmes animaux, ces variations sont très répétables d'une année sur l'autre (figure $4 \mathrm{~b}$ ).

Chez le bouc nain américain, la FSH est élevée au printemps (Muduuli et al 1979). Dans une autre race, au contraire, la concentration moyenne de FSH reste basse la majeure partie de l'année, mais présente un pic fin juillet, quand la LH est la plus élevée (Miyamoto et al 1987).

Les variations saisonnières de la $\mathrm{LH}$ sont commandées par des modifications de l'intensité de la rétroaction négative des stéroïdes, particulièrement de l'œstradiol $17 \beta$ : des boucs Cashmere australiens castrés, porteurs d'un implant sous-cutané qui libère une quantité constante d'œstradiol $17 \beta$, manifestent une variation saisonnière de $\mathrm{LH}$ parallèle à celle des animaux entiers, alors que les boucs castrés

\section{La sécrétion pulsatile de LH induit la libération de testostérone.}


Figure 3. Niveau de base, fréquence (a) et amplitude des pulses et concentrations moyennes (b) de $\mathrm{LH}$ et de testostérone plasmatique (c) chez 6 boucs Alpins (d'après Delgadillo et Chemineau 1992).

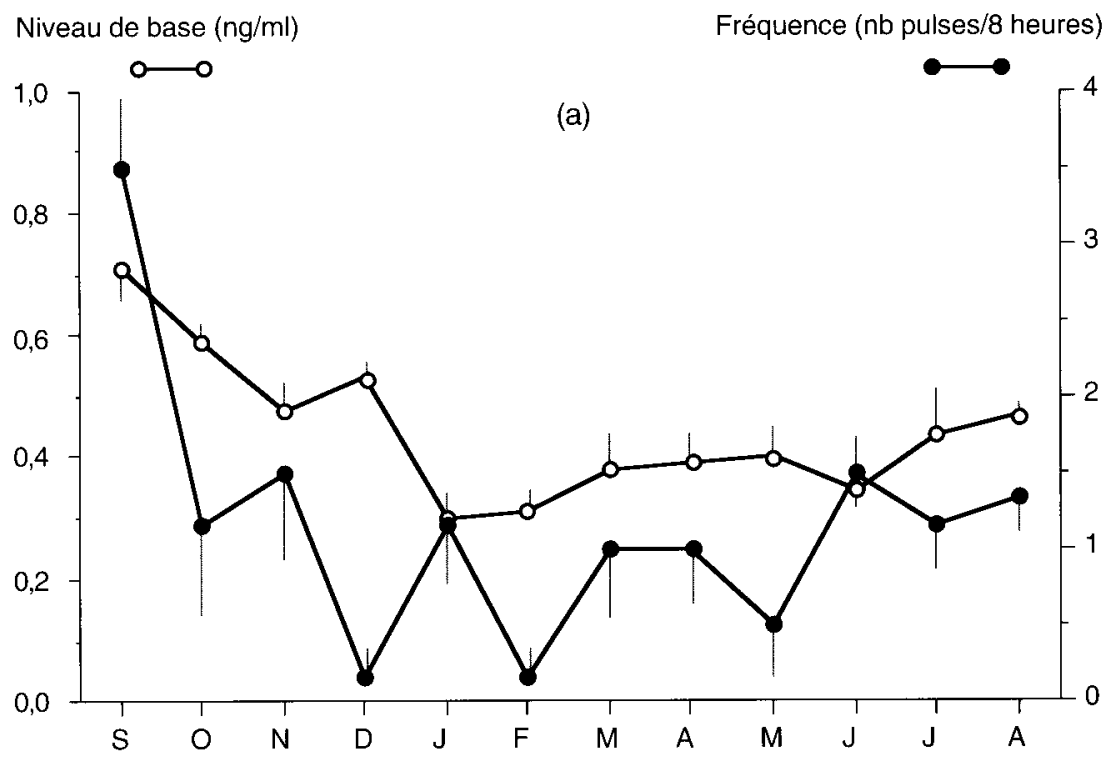

La fréquence et l'amplitude des pics de LH et la concentration de testostérone évoluent avec la saison : elles sont faibles de janvier $\grave{a}$ mai et augmentent jusqu'en aoûtseptembre.

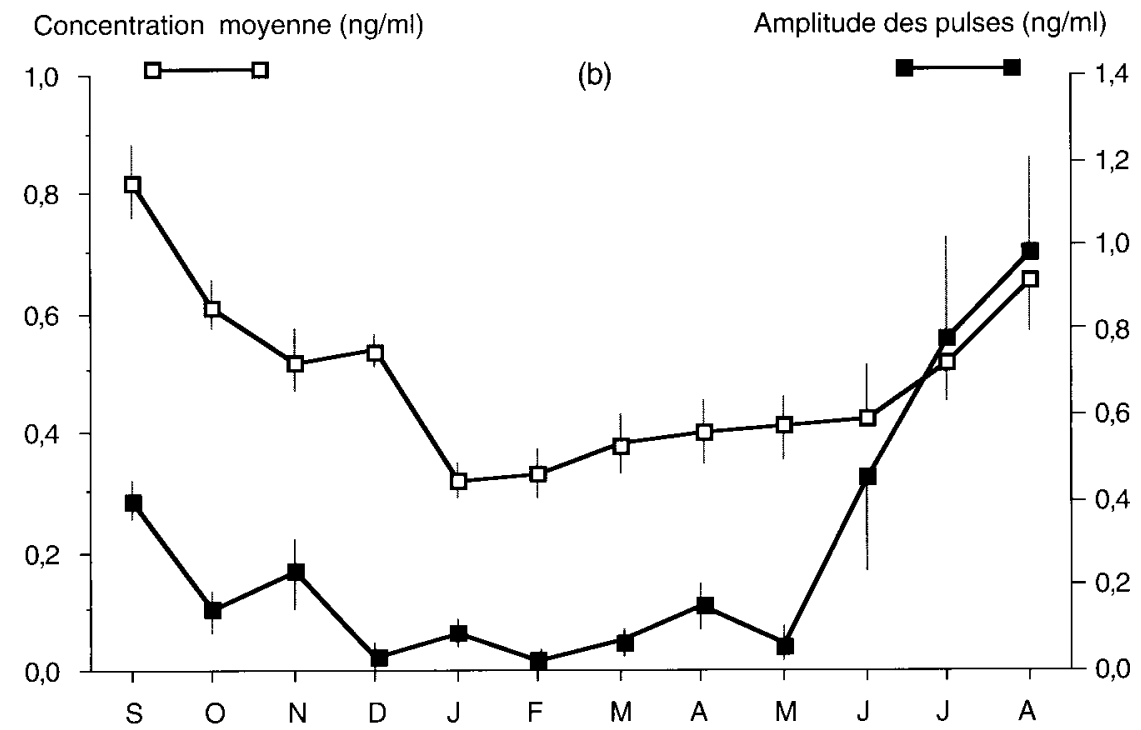

Testostérone plasmatique ( $\mathrm{ng} / \mathrm{ml}$ )

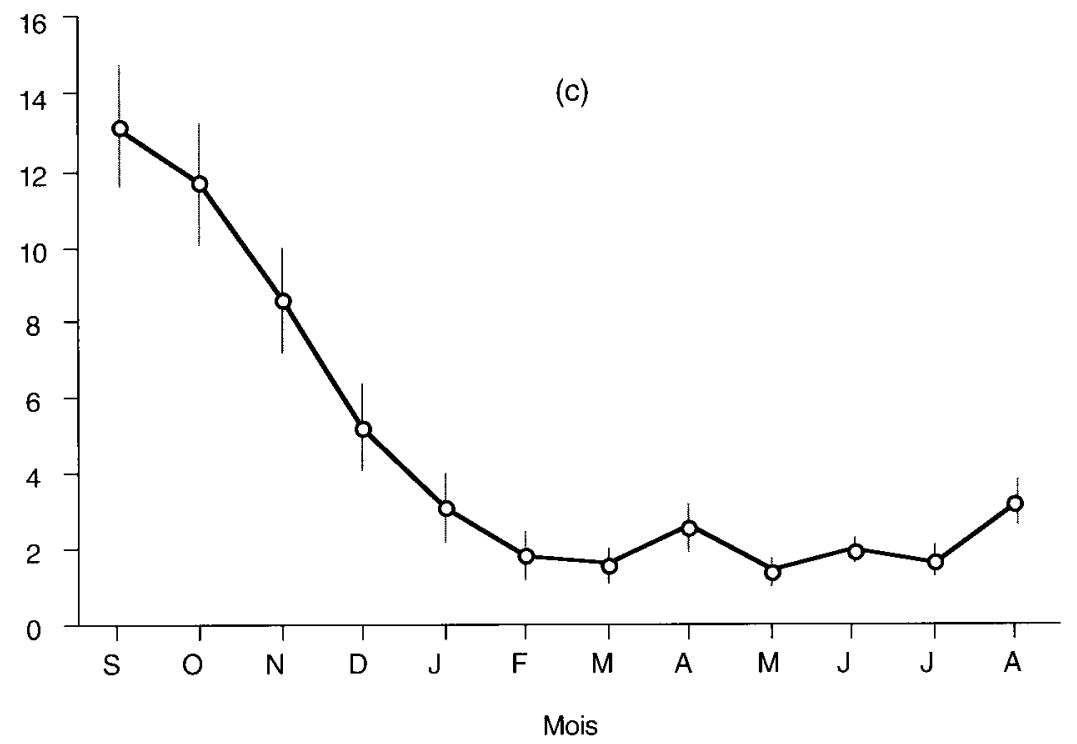


Figure 4. Poids testiculaire et comportement sexuel, mesuré par la latence à l'éjaculation (a), testostérone plasmatique (b) et motilité des spermatozoïdes (c), chez 6 boucs Alpins (d'après Delgadillo et al 1991, Delgadillo et Chemineau 1992).

Poids testiculaire $(\mathrm{g})$

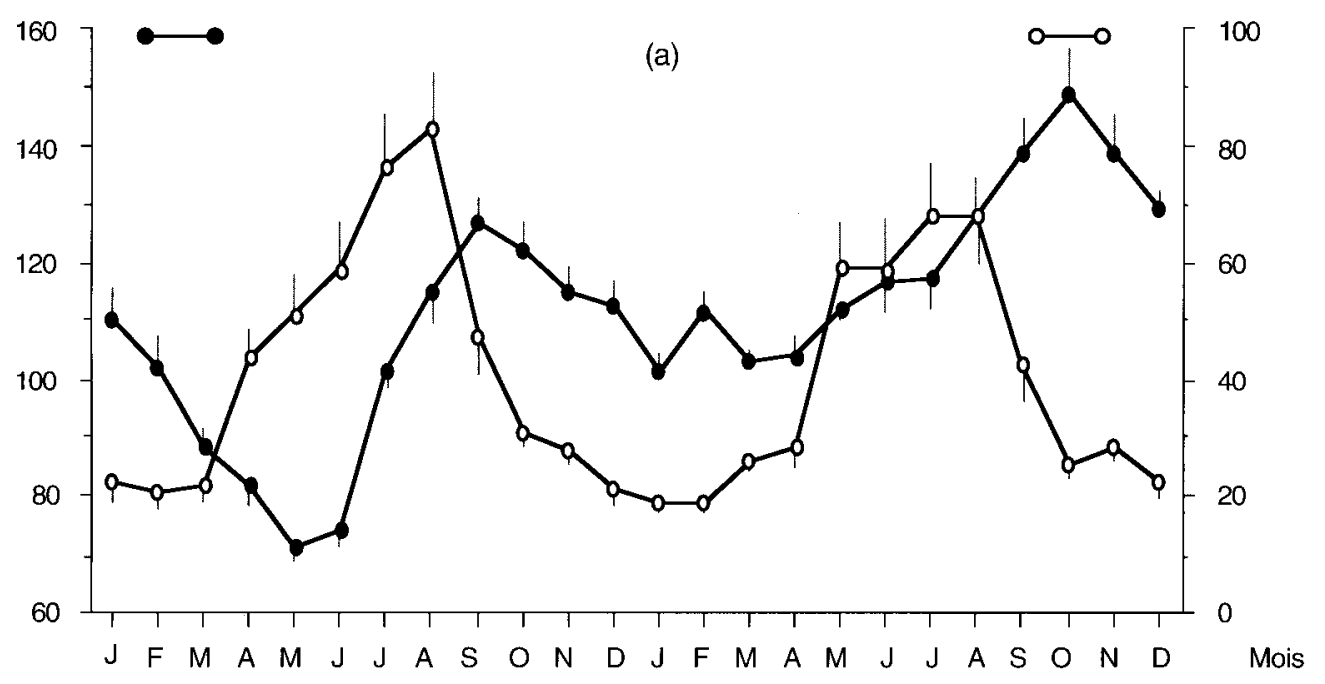

Testostérone plasmatique $(\mathrm{ng} / \mathrm{ml})$

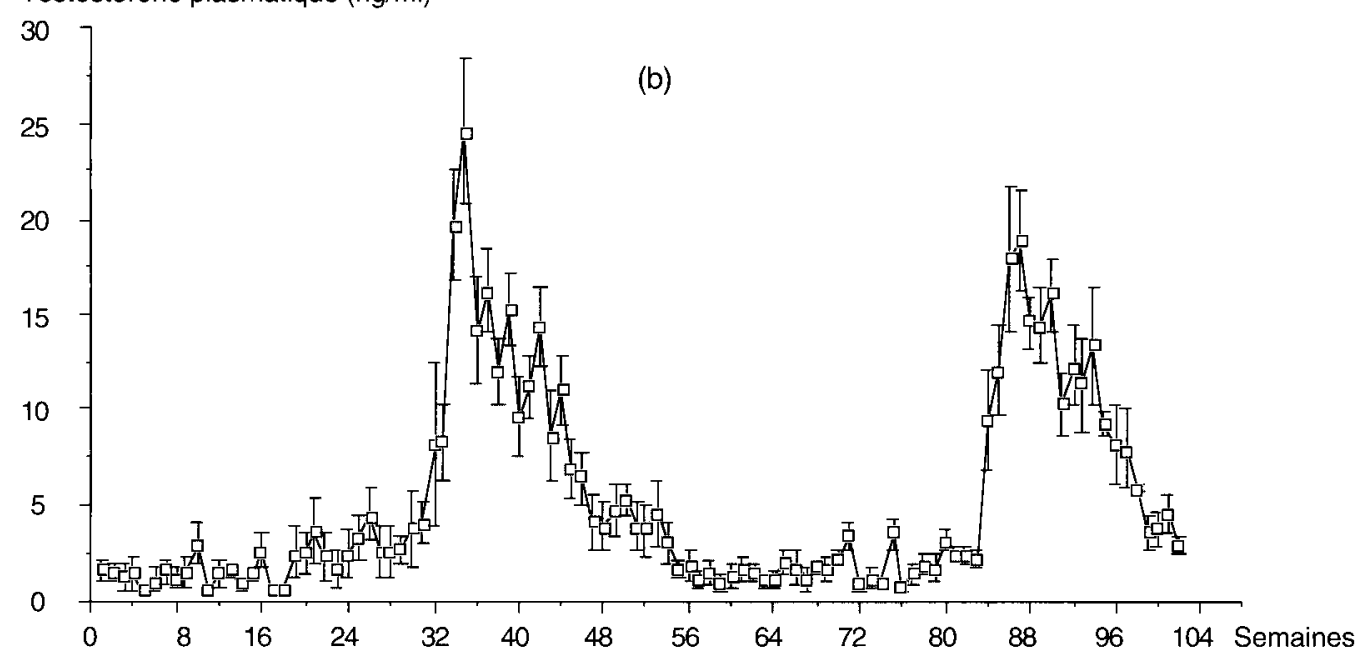

Motilité individuelle des spermatozoïdes (indice de 0 à 5)

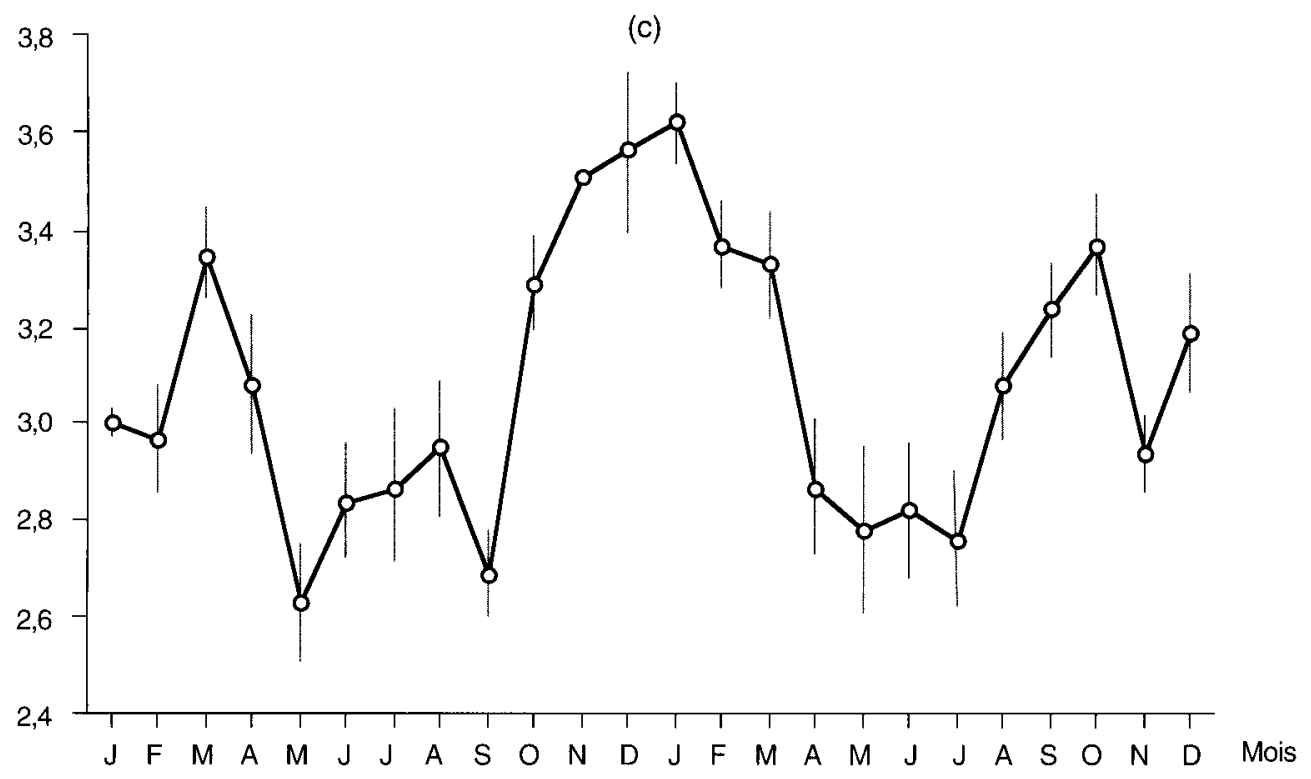

\section{Le poids} testiculaire, le comportement sexuel et la qualité de la semence évoluent parallèlement aux modifications hormonales. 
sans implant d'œstradiol ne montrent aucun changement au cours de l'année (WalkdenBrown 1991).

Un parallélisme très étroit entre les niveaux endocriniens, les changements du poids testiculaire (reflet de l'activité spermatogénétique) et le comportement sexuel (figure 4a), confirme que c'est bien l'activité neuroendocrinienne qui commande les variations saisonnières d'activité sexuelle chez le bouc. L'augmentation de l'activité pulsatile de la LH (amplitude en juin-juillet, fréquence en septembre) entraîne le début de la croissance testiculaire (juillet-août), puis la libération de la testostérone (septembre) qui stimule le comportement sexuel (augmentation du nombre de saillies par test de comportement, diminution de la latence à l'éjaculation) et la qualité de la semence (octobre) (figure $4 \mathrm{c}$ ).

La saison entraîne également des changements importants dans la prolactine plasmatique ; sa concentration est élevée au printemps et en été et basse en automne et en hiver (figure 5), ce qui conditionne, en partie, la croissance saisonnière et la mue du pelage (Gebbie 1993).

Enfin, la saison, par l'intermédiaire de la testostérone qui agit sur les glandes sébacées de la peau de la tête et du cou, modifie fortement l'odeur des boucs, plus forte pendant la saison sexuelle que pendant la saison d'ancestrus (Walkden-Brown 1991).

\section{3 / Contrôle photopériodique de l'activité neuroendocrinienne}

Chez les races saisonnées des milieux tempérés, c'est la durée quotidienne d'éclairement (photopériode) et ses variations qui commandent les changements saisonniers de l'activité neuroendocrinienne. La perception de la durée du jour se fait par la rétine qui transmet, par voie nerveuse, l'information à la glande pinéale (ou épiphyse). Cette dernière synthétise et sécrète la mélatonine dans la circulation générale, uniquement pendant l'obscurité. La durée de sécrétion au cours de 24 heures est donc directement corrélée à la durée de la nuit. Chez le bouc Alpin, à la latitude de $45^{\circ}$ Nord, la durée de sécrétion de mélatonine est longue en décembre ( 8 heures de lumière, 15 heures de sécrétion de mélatonine) et courte en juin (15 heures de lumière, 8,7 heures de sécrétion) (figure 6).

C'est par l'intermédiaire de cette durée de sécrétion que les animaux réagissent à la durée du jour et répondent aux variations photopériodiques. Chez des boucs Alpins placés dans un régime faisant alterner deux mois de jours longs (16 heures de lumière par 24 heures) et deux mois de jours courts (8 heures de lumière par 24 heures), l'activité testiculaire est modifiée sous l'influence de la photopériode. Les jours courts stimulent l'activité pulsatile de LH et les jours longs l'inhibent. Sous le contrôle de ces changements, le poids des testicules et leur activité endocrine (sécrétion de testostérone) présentent des alternances de hauts et faibles niveaux. La testostérone commence à s'élever dès la quatrième semaine après le début des jours courts et diminue au cours de la deuxième semaine après le début des jours longs (figure 7).

Les variations photopériodiques entraînent également celles de la sécrétion de prolactine. Une à deux semaines après le début des jours longs, les niveaux de prolactine s'élèvent, puis se maintiennent à un niveau élevé pendant ceux-ci. Inversement, une à deux semaines après le début des jours courts, la prolactine diminue et reste faible pendant ceux-ci (Delgadillo et Chemineau 1992).

Figure 5. Prolactine plasmatique chez 6 boucs Alpins (d'après Delgadillo et Chemineau 1992).

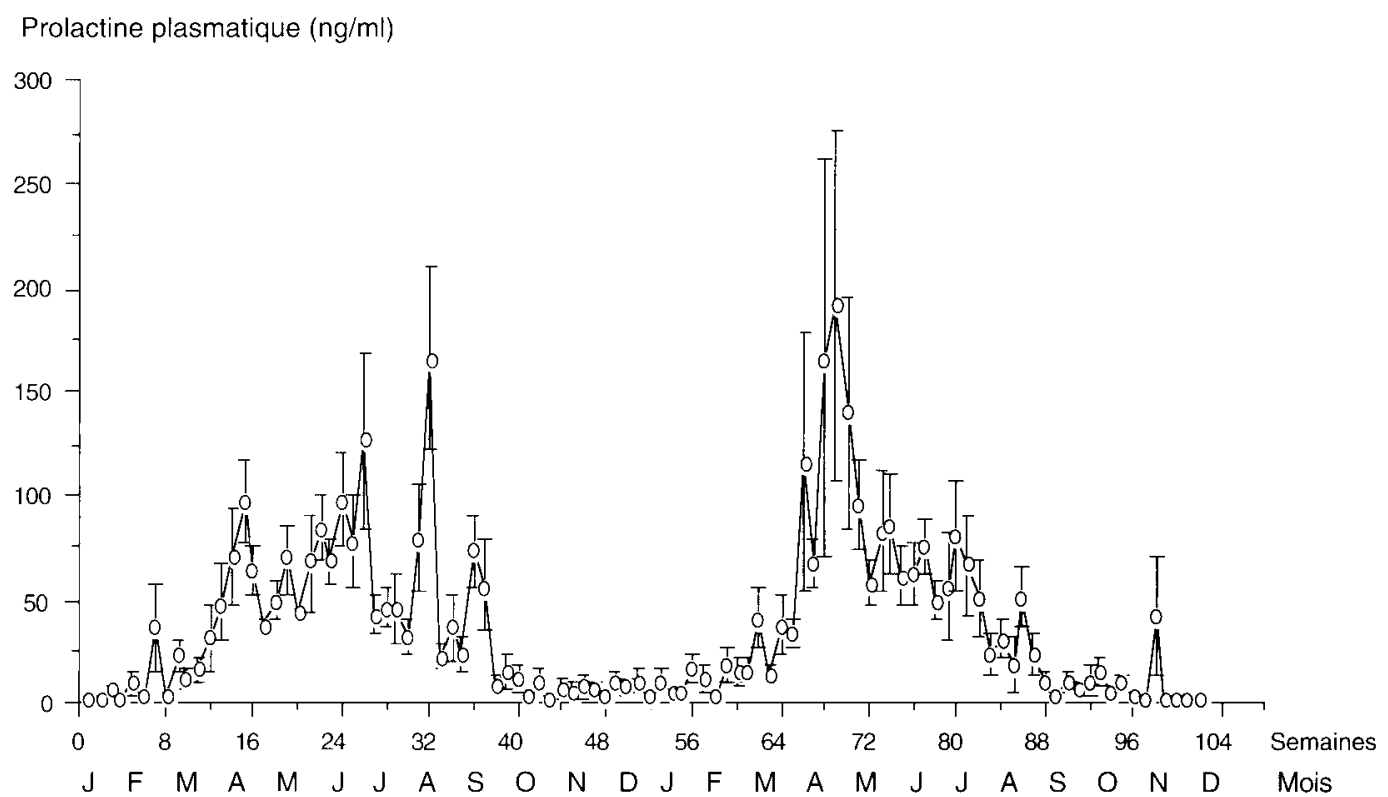




\section{4 / Interaction régime alimentaire et saison chez les mâles}

Les variations saisonnières de l'activité gonadotrope peuvent être modifiées par le régime alimentaire. Ainsi, chez des boucs Cashmere australiens recevant une ration à base de luzerne, l'activité pulsatile de LH augmente plus tôt et avec plus forte amplitude que chez les boucs maintenus avec une ration de pangola (herbe tropicale à faible teneur en matières azotées). En début de saison sexuelle, 7 pulses de LH sont observés en 8 heures chez les premiers, contre 2 seulement chez les seconds. Le nombre de pulses de testostérone, le poids testiculaire, l'activité des glandes sébacées et la "note d'odeur" des boucs sont également augmentés par le haut régime alimentaire (Walkden-Brown 1991).

Des boucs soumis à de tels régimes alimentaires contrastés possèdent un pouvoir d'induction différent de l'activité ovulatoire des femelles par "effet bouc". Il y a significativement plus de chèvres qui ovulent après la mise en contact avec des boucs soumis au régime haut, qu'après la mise en contact avec ceux recevant un régime alimentaire bas (71 vs 38\% au jour 5 après l'introduction du mâle, respectivement)(Walkden-Brown 1991).

\section{2 / Activité neuroendocrinienne de la femelle}

La chèvre non gestante manifeste des cycles œestriens et ovulatoires qui se succèdent à intervalles plus ou moins réguliers. De nom-
Figure 6. Mélatonine plasmatique chez 6 boucs Alpins, en décembre (jours courts) et en juin (jours longs) (d'après Delgadillo et Chemineau 1992).

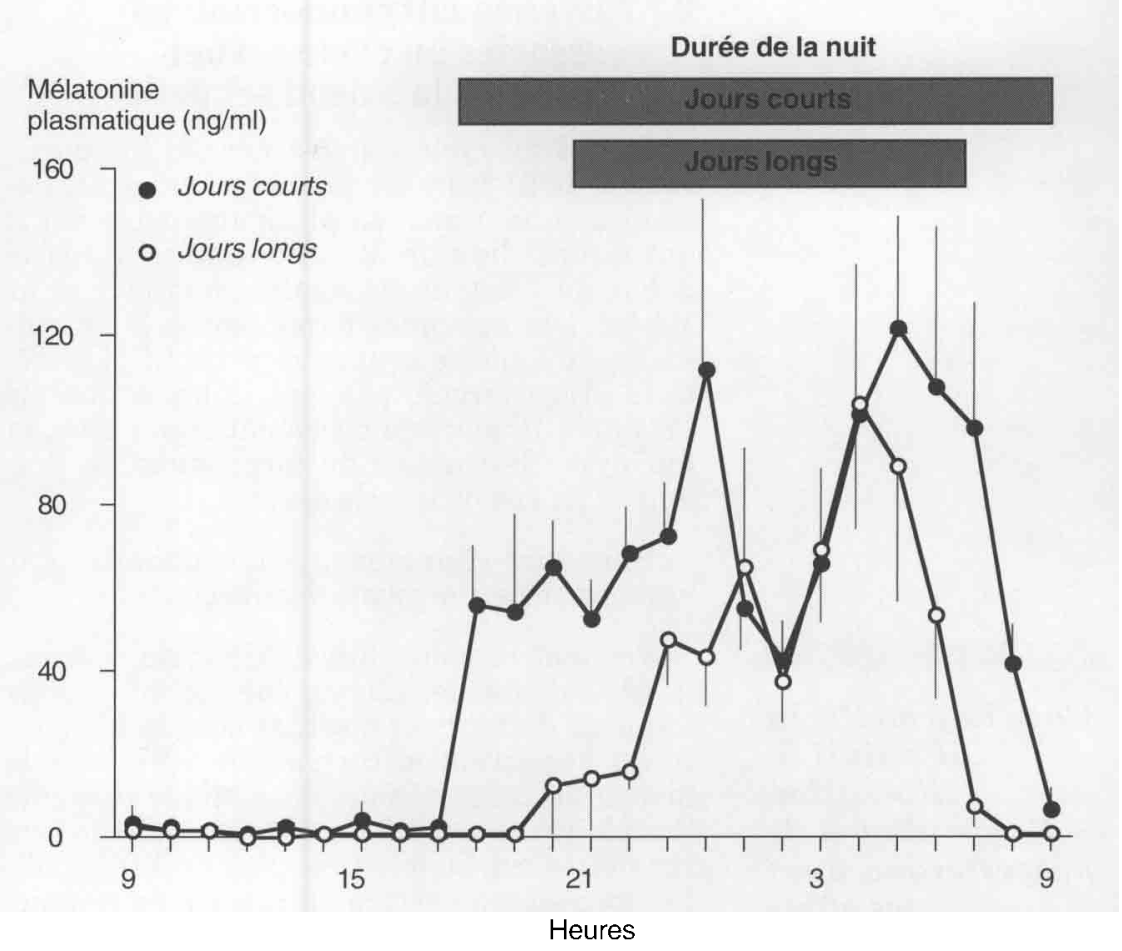

breux changements neuroendocriniens sont associés à cette cyclicité. Dans les races saisonnées, la cyclicité n'est pas permanente au cours de l'année, définissant ainsi une saison d'anœstrus et une saison sexuelle. L'activité neuroendocrinienne pendant l'anœstrus est

Figure 7. Testostérone plasmatique de 6 boucs Alpins soumis à une alternance de 2 mois de jours longs (16 heures de lumière/24) et 2 mois de jours courts ( 8 heures de lumière /24) (d'après Delgadillo et Chemineau 1992).

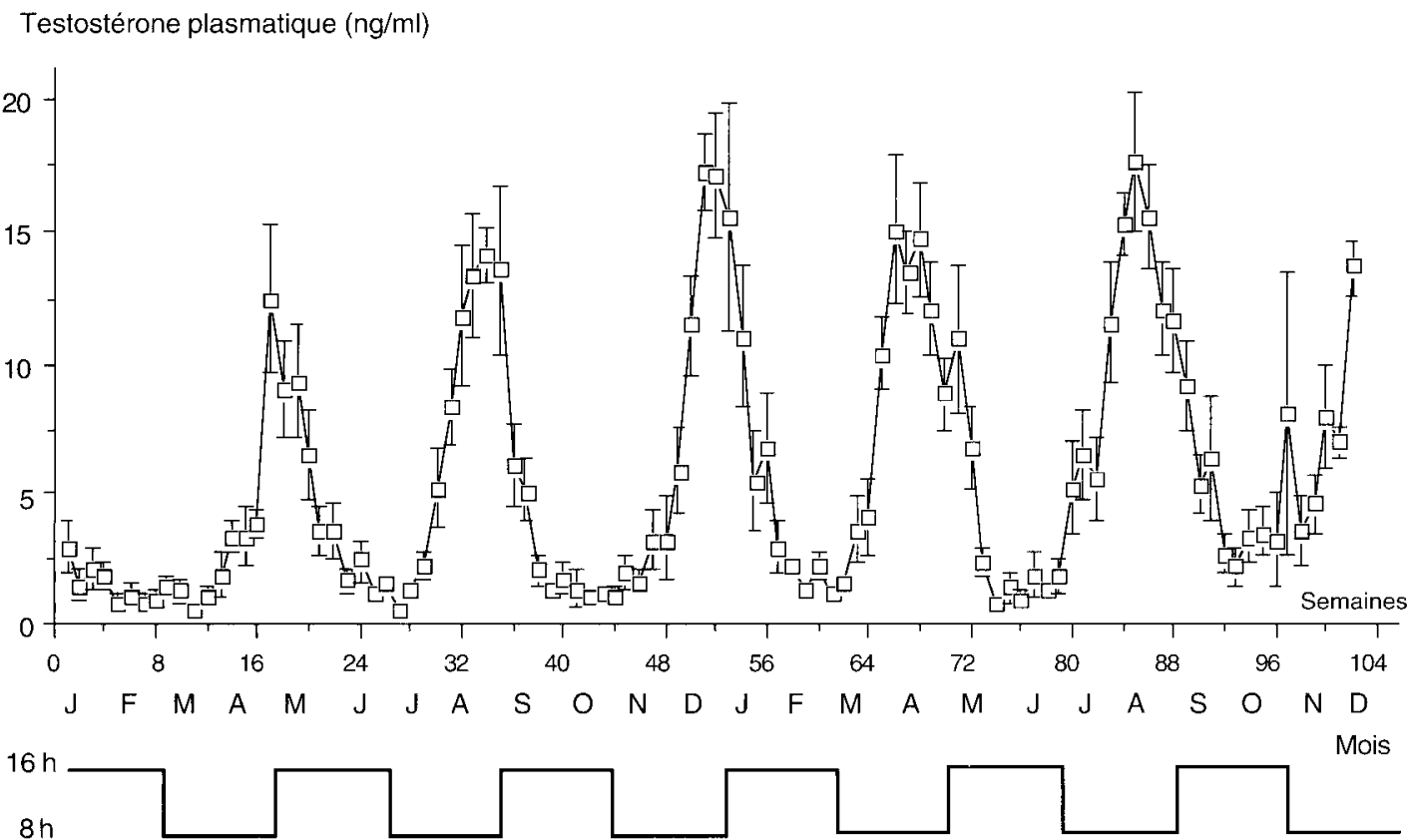

Photopériode 
évidemment très différente de celle observée pendant la saison sexuelle.

\section{1 / Evénements endocriniens associés au cycle sexuel pendant la saison sexuelle}

Quand un cycle œstrien normal (de durée voisine de 21 jours) se produit, il est généralement associé à une (ou plusieurs) ovulation(s) qui a (ont) lieu de 30 à 36 heures après le début de l'œstrus (Gonzalez-Stagnaro et al 1984a). Le corps jaune formé issu de la lutéinisation du follicule ovulatoire est actif (il sécrète la progestérone) pendant la phase lutéale (de durée 16 jours en moyenne). Après quoi, la lutéolyse (destruction du corps jaune) se produit et un nouveau cycle débute.

Plusieurs changements hormonaux sont associés aux événements décrits ci-dessus.

Pendant la phase lutéale du cycle ostrien, la LH est libérée, comme chez le mâle, sous forme de décharges pulsatiles dont la fréquence est négativement corrélée $(r=-0,97)$ avec le niveau de progestérone plasmatique d'origine lutéale (Sutherland 1987a). L'amplitude de ces pulses est limitée (pas plus de $1,0 \mathrm{ng} / \mathrm{ml}$ ). La progestérone exerce un rôle clé de rétroaction négative dans la régulation de la $\mathrm{LH}$ au cours du cycle. Les quantités circulantes dans le sang périphérique doivent cependant être suffisantes pour exercer un rétrocontrôle efficace (Sutherland $1987 \mathrm{~b}$, Chemineau et al 1988).

Aux alentours des jours $16-17$ du cycle $(\mathrm{J} 0=$ jour de l'œstrus), les prostaglandines utérines, peut-être sous l'influence de l'ocytocine ovarienne (Homeida 1986), provoquent la lutéolyse (Horton et Polyser 1976). Immédiatement après celle-ci, la brusque diminution de la progestérone entraîne une forte augmentation de la fréquence de décharge des pulses de LH et de leur amplitude (Mori et Kano 1984, Sutherland 1987a). Cette augmentation de l'activité gonadotrope provoque une stimulation de la croissance des follicules de diamètre supérieur à $1,0 \mathrm{~mm}$ (Akusu et al 1986) et de leur activité stéroidogène (Kanai et Ishikawa 1988). Ils sécrètent alors l'œstradiol 17ß en quantités croissantes (Mori et Kano 1984) : le niveau plasmatique s'élève de 10 à $30 \mathrm{pg} / \mathrm{ml}$ dans les trois jours précédant l'œstrus (figure 8a)(Bono et al 1983).

Le niveau croissant et élevé d'œstradiol 17ß déclenche alors le comportement d'œstrus. Il faut remarquer que la chèvre, contrairement à la brebis, ne nécessite pas une imprégnation préalable par la progestérone pour que l'œstradiol puisse induire le comportement d'œestrus ; l'œstradiol seul est suffisant (Sutherland $1987 \mathrm{c})$. Ceci explique que, au contraire de la brebis, la saison sexuelle des chèvres commence souvent par un comportement d'œstrus sans ovulation silencieuse préalable, voire même par un œstrus sans ovulation (Chemineau et al 1992). Cela explique également que, lors de la réponse à "l'effet bouc" des œestrus soient associés à la première et à la seconde ovulation induite, ce qui n'est jamais le cas chez la bre- bis (Chemineau 1989, brebis : Martin et al 1986)

Au cours du cycle, l'élévation d'œstradiol $17 ß$ dans la circulation générale induit également, par rétroaction positive (Dial et al 1985), une décharge massive de LH par l'hypophyse : c'est le pic préovulatoire (figure $8 \mathrm{~b}$ ). Il dure de 8 à 10 heures et son niveau dépasse $50 \mathrm{ng} / \mathrm{ml}$ de plasma. Le maximum du pic est atteint 3 heures après le maximum d'œstradiol $17 \beta$ et 10 à 15 heures après le début de l'œstrus (Chemineau et al 1982, Pelletier et al 1982, Mori et Kano 1984, Gonzalez-Stagnaro et al $1984 a$, Sutherland 1987a). La FSH est également libérée massivement en même temps que la $\mathrm{LH}$ et pour la même durée (figure $8 \mathrm{~b}$ ). Un second pic de FSH, de durée plus longue que le premier, est observé ou non, 48 heures après le premier (Chemineau et al 1982, Bono et al 1983).

La prolactine évolue également de façon importante : un premier pic est observé dans les 24 heures qui précèdent l'œestrus, puis une décharge massive se produit en même temps que le pic préovulatoire de LH (figure 8c) (Chemineau et al 1982, Gonzalez-Stagnaro et al 1984b).

La décharge préovulatoire de gonadotropines provoque la lutéinisation du follicule et l'arrêt de la sécrétion d'œstradiol. Dans les 12 heures qui suivent le pic, le niveau plasmatique de celle-ci est revenu à son niveau de base de $8 \mathrm{pg} / \mathrm{ml}$ (Chemineau et al 1982). Les mécanismes de transformation des cellules folliculaires, conduisent alors à l'ovulation qui se produit environ 20 heures après le pic préovulatoire de LH (Gonzalez-Stagnaro et al 1984a). Le follicule se transforme alors en corps jaune et se met à sécréter la progestérone, en partie au moins sous l'influence de la LH dont l'activité pulsatile est élevée (4 à 7 pulses en 8 heures) jusqu'au jour 7 du cycle où la fréquence se stabilise aux environs de 1,5 pulses en 8 heures (Sutherland 1987a). C'est le milieu de la phase lutéale, un nouveau cycle commence.

Les événements endocriniens qui viennent d'être rapportés ont été décrits lors d'œstrus naturels ou qui suivent une lutéolyse induite par des prostaglandines. Dans le cas d'une induction d'cestrus et d'ovulation par un traitement hormonal utilisant une substance gonadostimulante (PMSG), l'ensemble des éléments de la séquence sont présents avec cependant quelques différences. La stimulation folliculaire est immédiate après l'injection de PMSG, puisque les niveaux maximums, en général plus élevés, sont atteints 18 heures après l'injection. Le déclenchement du pic préovulatoire est plus tardif ( 23 heures de plus entre maximum d'œstradiol $17 \beta$ et maximum de LH) et il n'existe plus de second pic de FSH 48 heures après le premier (Chemineau et al 1982). Par ailleurs, malgré un intervalle plus long entre maximum d'œstradiol $17 \beta$ et pic préovulatoire de $\mathrm{LH}$, la décharge préovulatoire se produit plus tôt après le début des chaleurs $(5,6$ vs 15,7 heures) et l'intervalle pic de LH-ovulation est légèrement plus long (4 heures de plus) (Gonzalez-Stagnaro et al 1984a). 
Figure 8. Evénements endocriniens associés au déroulement du cycle oestrien chez la chèvre : (a) variations d'cestradiol $17 B$ et de progestérone, (b) variations de $L H$ et $F S H$, (c) variations de prolactine. L'échelle des temps débute au moment du maximum de concentration de la LH pendant le pic préovulatoire (d'après Chemineau et al 1982).

Progestérone plasmatique $(\mathrm{ng} / \mathrm{ml})$

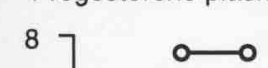

$$
6
$$

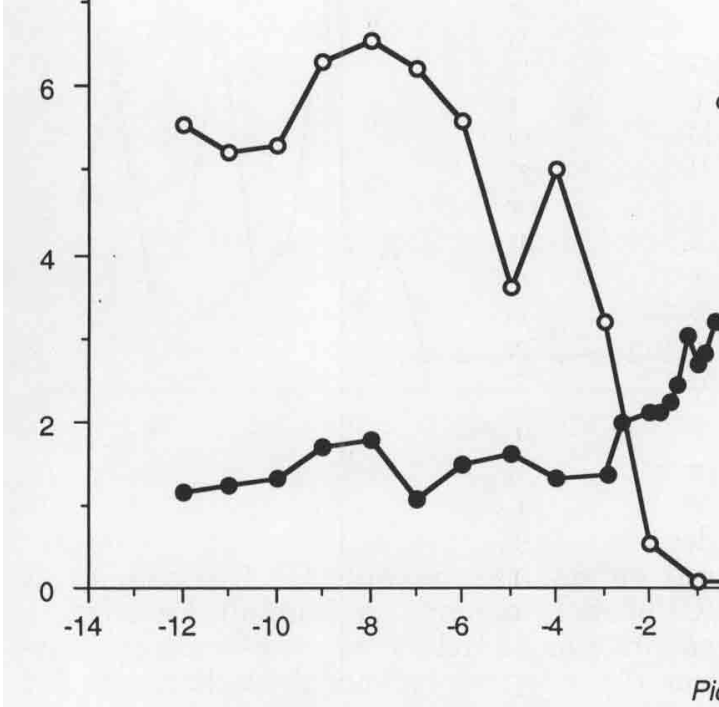

(b)

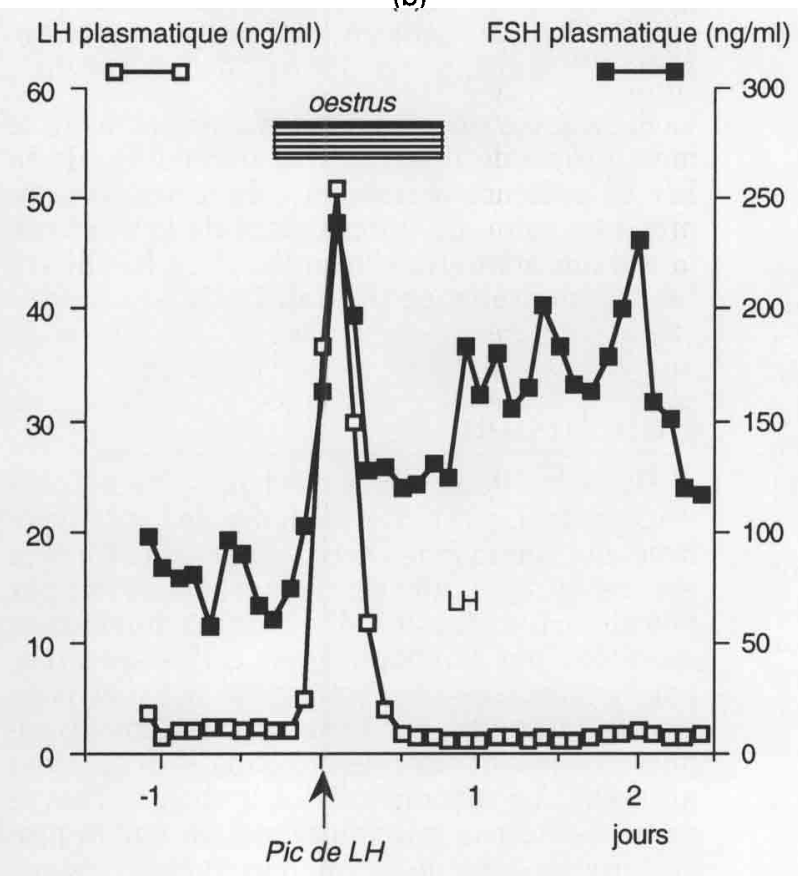

\section{2 / Activité neuroendocrinienne pendant l'anœestrus}

Chez les races saisonnées, la saison d'ancestrus et d'anovulation se caractérise par une absence quasi-totale de cycles. On observe également une faible fréquence de décharge des pulses de LH (moins de 2 pulses en 6 heures, début août), alors qu'il n'y a pas de progestérone endogène. La fréquence et l'amplitude augmentent à l'approche de la saison sexuelle : plus de 3 pulses en 6 heures à la mi-septembre (Chemineau et al 1988). (a) Oestradiol $17 \beta$ plasmatique $(\mathrm{pg} / \mathrm{ml})$
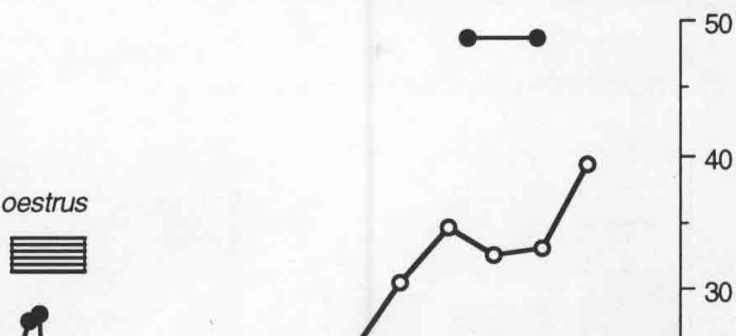

$-20$

(c)

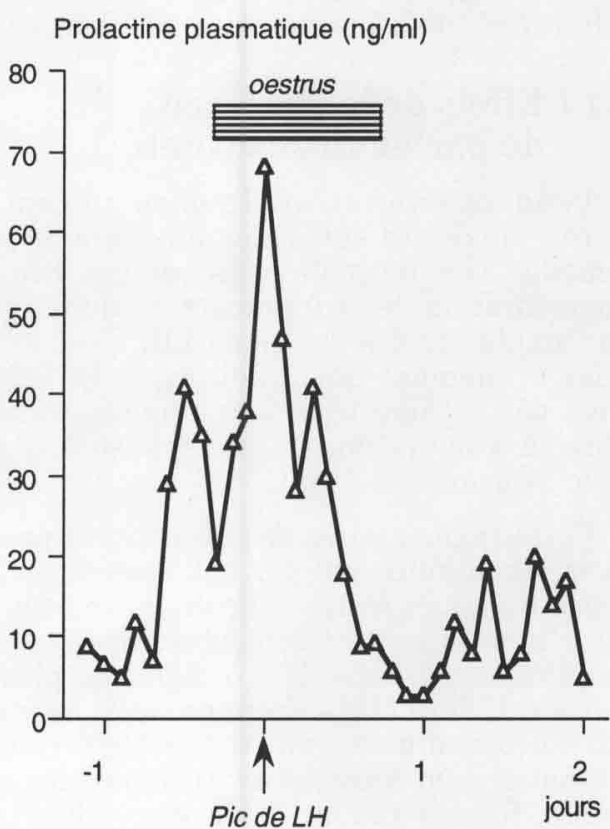

La faible activité LH pendant l'anœstrus est due à une rétroaction négative forte de l'œstradiol $17 ß$ sur l'axe hypothalamo-hypohysaire. La présence d'un implant d'œstradiol chez la chèvre castrée provoque, pendant la saison d'anœstrus et non pendant la saison sexuelle, une diminution de la fréquence des pulses de LH (de 9 à 4 pulses en 6 heures ; figure 9) (Sutherland 1987b).

Cette augmentation saisonnière de la rétroaction négative de l'œstradiol est, exactement comme chez le bouc, sous le contrôle de la pho-
L'importance des rétroactions négatives des hormones ovariennes varie au cours de l'année, en relation directe avec la durée du jour. 
Figure 9. Nombre de pulses de $\mathrm{LH}$ en 6 heures de prélèvement, chez des chèvres castrées de race Saanen pendant la saison sexuelle (SS) ou pendant l'anoestrus (AN) et portant (+E2) ou non (-E2) un implant sous-cutané d'oestradiol (4 chèvres par groupe - d'après Chemineau et al 1988).

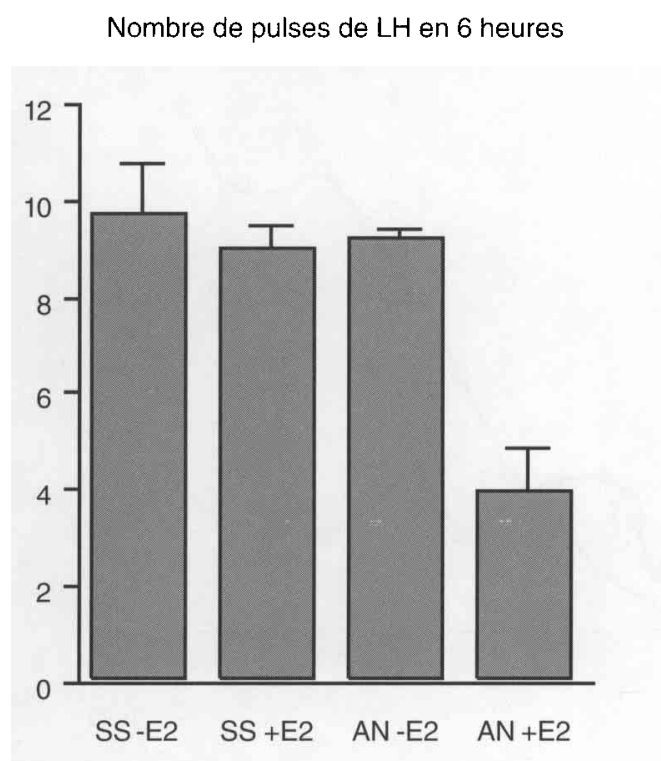

topériode, par l'intermédiaire de la mélatonine (Mori et al 1987, Chemineau et al 1988).

\section{3 / Effets de la présence de partenaires sexuels}

Pendant l'anœstrus, la mise en contact, après plusieurs semaines d'isolement, des femelles avec un mâle provoque une brusque augmentation de la fréquence de décharge et de l'amplitude des pulses de LH. C'est le premier événement qui conduira, si la femelle n'est pas en anœstrus trop intense, à une ou plusieurs ovulation(s), en réponse à "l'effet bouc" (Chemineau 1989).

Cette augmentation de pulsatilité se produit dans les minutes qui suivent la mise en présence du bouc. Avant celle-ci, 0,3 pulses par femelle en 3 heures sont observés avec une amplitude moyenne de $0,5 \mathrm{ng} / \mathrm{ml}$ de plasma. Pendant les trois heures qui suivent, 2,2 pulses d'une amplitude moyenne de $1,2 \mathrm{ng} / \mathrm{ml}$ sont enregistrés (Chemineau et al 1986b, Restall 1992b). Un exemple illustrant ce phénomène chez la chèvre Saanen est représenté sur la figure 10 . Chez la chèvre Créole de Guadeloupe, 80 \% des femelles répondent dans les 80 minutes qui suivent l'introduction $\mathrm{du}$ bouc en présentant un pulse de LH d'une amplitude moyenne de $1,7 \mathrm{ng} / \mathrm{ml}$. Cette augmentation de l'activité hypohysaire stimule la croissance folliculaire et provoque l'apparition d'un pic préovulatoire de LH qui va induire l'ovulation (53 heures après la mise en présence du bouc avec la chèvre Créole) (Chemineau et al 1986a).

Dans les conditions normales, la perception du mâle se fait par la voie olfactive, sans que ce soit toutefois l'odeur caractéristique du bouc
Figure 10. Augmentation de la fréquence des pulses de $\mathrm{LH}$ chez une chèvre Saanen après la mise en présence du bouc (d'après Chemineau et al 1986a).

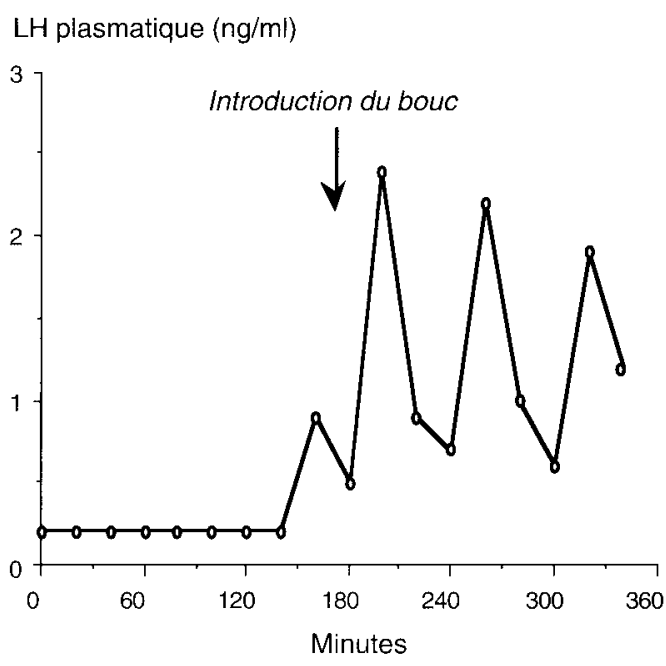

qui en soit responsable (P. Chemineau et J. Cohen-Tannoudji, non publié). En effet, la suppression de l'odorat ne modifie pas l'apparition d'une forte fréquence de décharge de $\mathbf{L H}$ dans les minutes qui suivent la mise en contact avec le mâle, ce qui semble indiquer que les femelles utilisent une autre voie sensorielle (Chemineau et al 1986a, Restall 1992b).

Si la mise en contact des chèvres avec le bouc provoque une réponse immédiate de la LH, la présence permanente de boucs vasectomisés entraîne un allongement de la durée de la saison sexuelle annuelle chez la chèvre "Feral" australienne (Restall 1992a).

\section{Conclusion}

Dans les deux sexes c'est le système nerveux central, par l'intermédiaire de l'antéhypohyse, qui commande l'activité sexuelle. Chez la chèvre, la régulation du cycle sexuel se fait par l'équilibre entre les différentes hormones sécrétées par l'hypophyse et celles sécrétées par l'ovaire. Les rétroactions négative et positive des stéroïdes sur l'axe hypothalamo-hypophysaire jouent un rôle clé dans la régulation du cycle. Au contraire de la brebis, la chèvre ne nécessite pas une imprégnation par la progestérone pour que le comportement d'œestrus puisse être induit par l'œestradiol.

Les modifications dues à la saison ou à la présence de partenaires sexuels, agissent également au niveau du système nerveux central. La modulation de la rétroaction négative de l'œstradiol sur la LH par la photopériode est le mécanisme responsable de la faible activité neuroendocrinienne pendant l'anœstrus.

\section{Remerciements}

Les auteurs souhaitent remercier Agnès Daveau, Françoise Maurice, Pierre et Robert Pignon, le laboratoire de dosages hormonaux de Nouzilly, ainsi que les nombreuses personnes ayant participé aux prélèvements sanguins. 


\section{Références bibliographiques}

Akusu M. O., Osuagwuh, A. I. A., Akpokodje, J. U., Egbunike, G. N., 1986. Ovarian activities of the West African dwarf goat (Capra hircus) during oestrus. J. Reprod. Fert., 78, 459-462.

Bono G., Cairoli F., Tamanini C., Abrate, L., 1983. Progesterone, estrogen, LH, FSH and PRL concentrations in plasma during the estrous cycle in goat. Reprod. Nutr. Dévelop., 23, 217-222.

Buttle H.L., 1974. Seasonal variation of prolactin in plasma of male goats. J. Reprod. Fert., 37, 95-99.

Chemineau P., 1989. L'effet bouc : mode d'action et efficacité pour stimuler la reproduction des chèvres en anoestrus. INRA Prod. Anim., 2, 97-104.

Chemineau P., Gauthier D., Poirier J. C., Saumande J., 1982. Plasma levels of LH, FSH, Prolactin, Oestradiol 17ß and progesterone during natural and induced oestrus in the dairy goat. Theriogenology, 17, 313-323.

Chemineau P., Lévy F., Thimonier J., 1986a. Effects of anosmia on LH secretion, ovulation and oestrous behaviour induced by males in the anovulatory Creole goat. Anim. Reprod. Sci., 10, 125-132.

Chemineau P., Normant E., Ravault J.P., Thimonier J., 1986b. Induction and persistence of pituitary and ovarian activity in the out-of-season lactating dairy goat after a treatment combining a skeleton photoperiod, melatonin and the male effect. J. Reprod. Fert., 78, 497-504.

Chemineau P., Martin G.B., Saumande J., Normant E., 1988. Seasonal and hormonal control of pulsatile LH secretion in the dairy goat (Capra hircus). J. Reprod. Fert., 88, 91-98.

Chemineau P., Daveau A., Maurice F., Delgadillo J. A., 1992. Seasonality of oestrus and ovulation is not modified by subjecting female Alpine goats to a tropical photoperiod. Small Ruminant Research, 8 , 299-312.

Delgadillo J.A., Chemineau P., 1992. Abolition of the seasonal release of luteinizing hormone and testosterone in Alpine male goats (Capra hircus) by short photoperiodic cycles. J. Reprod. Fert., 94, 4555 .

Delgadillo J.A., Leboeuf B., Chemineau P., 1991. Decrease in the seasonality of sexual behaviour and sperm production in bucks by exposure to short photoperiodic cycles. Theriogenology, 36, 755-770.

Dial G. D., Wiseman B. S., Ott R. S., Smith A. L., Hixon J. E., 1985. Absence of sexual dimorphism in the goat : induction of luteinizing hormone discharge in the castrated male and female and in the intersex with estradiol benzoate. Theriogenology, 23, 351360 .

Gebbie F., 1993. Control of seasonal breeding and coat development in the goat. PhD Thesis, Univ Surrey, UK, 205 pp.

Gonzalez-Stagnaro C., Pelletier J., Cognié Y., Locatelli A., Baril G., Corteel J. M., 1984a. Descarga preovulatoria de LH y momento de ovulacion en cabras lecheras durante el celo natural o inducido por via hormonal. Proc. 10 th Intern. Congr. Anim. Reprod. \& A.I., Urbana, Ill. (USA), Vol. II, Comm. $\mathrm{N}^{\circ} 10$.
Gonzalez-Stagnaro C., Ravault J. P., Baril G., Corteel J. M., 1984b. Prolactinemia en la cabra durante el celo natural o inducido en periodo de anestro estacional. Proc. 10 th Intern. Congr. Anim. Reprod. \& A.I., Urbana, Ill. (USA), Vol. II, Comm. $\mathrm{N}^{\circ} 8$.

Homeida A. M., 1986. Role of oxytocin during the oestrous cycle of ruminants with particular reference to the goat. Anim. Breed. Abst., 54, 263268.

Horton E.W., Polyser L.N., 1976. Uterine luteolytic hormone : a physiological role for Prostaglandin F2 $\alpha$. Physiol.Rev., 56, 595-651.

Kanai Y., Ishikawa N., 1988. Pulsatile secretion of luteinizing hormone and plasma levels of ovarian steroids during the estrous cycle in the Shiba goat. Jpn. J. Anim. Reprod., 34, 105-110.

Martin G. B., Oldham C. M., Cognié Y., Pearce D. T., 1986. The physiological responses of anovulatory ewes to the introduction of rams. Livest. Prod. Sci., $15,219-247$.

Miyamoto A., Umezu M., Hamano K., Masaki J., 1987. Seasonal changes in inhibin activity in seminal plasma and serum concentrations of FSH, $\mathrm{LH}$ and testosterone in the male goat (Capra hircus). Theriogenology, 28, 67-76.

Mori Y., Kano Y., 1984. Changes in plasma concentrations of $\mathrm{LH}$, progesterone and oestradiol in relation to the occurrence of luteolysis, oestrus and time of ovulation in the Shiba goat (Capra hircus). J. Reprod. Fert., 72, 223-230.

Mori Y., Tanaka M., Maeda K., Hoshino K., Kano Y., 1987. Photoperiodic modification of negative and positive feedback effects of oestradiol on $\mathrm{LH}$ secretion in ovariectomized goats. J. Reprod. Fert., $80,523-529$.

Muduuli D. S., Sanford L. M., Palmer W. M., Howland B. E., 1979. Secretory patterns and circadian and seasonal changes in luteinizing hormone, follicle stimulating hormone, prolactin and testosterone in the male pygmy goat. J. Anim. Sci., $49,543-553$.

Pelletier J., Gonzalez-Stagnaro C., Baril G., Corteel J. M., 1982. La décharge préovulatoire de LH induite chez la chèvre en période d'anoestrus saisonnier. C. R. Acad. Sci., Paris, 294, Sér. III, 867 870 .

Restall B. J., 1992a. Seasonal variations in reproductive activity in Australian goats. Anim. Reprod. Sci., 27, 305-318.

Restall H., 1992b. Factors affecting ovulatory activity in the Australian Cashmere goat. PhD Thesis, Univ. Queensland, Australie, $190 \mathrm{pp}$.

Saumande J., Rouger Y., 1972. Variations saisonnières des taux d'androgènes dans le plasma de sang périphérique chez le bouc. C. R. Acad. Sci., Paris, 274, Sér. III, 89-92.

Sutherland S. R. D., 1987a. Progesterone concentration and pulsatile LH secretion during normal oestrous cycles in Angora-cross does. Proc. 4th AAAP Animal Science Congress, Hamilton, New Zealand, Feb 1-6, p.246. 
Sutherland S. R. D., 1987b. Effects of oestradiol and progesterone on LH secretion during anoestrus and the breeding season in ovariectomized Angora-cross does. Proc. 4th AAAP Animal Science Congress, Hamilton, New Zealand, Feb 1-6, p.230.

Sutherland S. R. D., 1987c. Progesterone and estrogen requirements for oestrous behaviour in goats and sheep. Proc. 4th AAAP Animal Science Congress, Hamilton, New Zealand, Feb 1-6, p.227.
Walkden-Brown S. W., 1991. Environmental and social influences on reproduction in Australian Cashmere goats. PhD Thesis, Univ Queensland, Australie, $237 \mathrm{pp}$.

\section{Summary}

Reproductive neuroendocrinology in the caprine species.

Hypothalamic activity commands the episodic discharge of gonadotropins in the general circulation. Pulses of LH, secreted by the pituitary and defined with their frequency and amplitude, stimulate the release of testosterone by the testis in the male and of estradiol and progesterone by the ovary in the female. FSH appears to be secreted more continuously in a non episodic manner. In the female goat, modulation of the negative and positive feed-back of progesterone and estradiol on the hypothalamo-pituitary axis plays a key role in the regulation of the differents events of the oestrous cycle. Progesterone priming is not necessary for the induction of estrous behaviour by estradiol.
In both sexes, seasonality of neuroendocrine activity is responsible for the large seasonal variations in sexual activity. Enhancement of the nega. tive feed-back of estradiol on the hypothalamo. pituitary axis is responsible for the low gonadotro. pin activity during anestrus. This effect is media ted by the photoperiod which acts on the central nervous system by the modification of the dura. tion of night-time melatonin secretion. Changes in the feeding regime also cause modifications in the gonadotropic activity.

Presence of males causes an immediate increase in the frequency of $\mathrm{LH}$ pulses in the females.

CHEMINEAU P., DELGADILLO J.A., 1994. Neuroendocrinologie de la reproduction chez les caprins. INRA Prod. Anim., 7 (5), 315-326. 